\title{
Characterizing complex metal-CFRP-hybrid structures by thermography
}

\author{
by M. Schwarz a,b, A. Hella and H.-G. Herrmannª,b \\ a Chair for Lightweight Systems, Saarland University, Campus E3 1, 66123 Saarbrücken, Germany, \\ Michael.schwarz@izfp.fraunhofer.de \\ b Fraunhofer IZFP Institute for Nondestructive Testing, Campus E3 1, 66123 Saarbrücken, Germany
}

\begin{abstract}
In this paper results of non-destructive testing of metal-CFRP-hybrid structures are shown. The hybrid samples vary from $2 \mathrm{D}$ to $3 \mathrm{D}$ geometry. As NDT-method active thermography with its excitation modes flash and induction through an eddy current coil are used. It is shown that artificial induced defects like pleats in the CFRP-layer and mechanically induced delaminations at the interface CFRP/thermoplastic can be detected. For the latter defect, a different damage behavior can be shown depending on the geometry.
\end{abstract}

\section{Introduction}

To fulfil today's legal requirements concerning $\mathrm{CO}_{2}$-emissions lightweight design can be a determining factor to reach the goals. Therefore, materials with a high lightweight potential like CFRP are widely used. As CFRP is a quite expensive material, it is not economical to design structures only of CFRP. Instead, a combination of several materials a so-called multi-material design can use the advantages of each material with low cost. In this paper a metal-CFRP-hybrid structure with a thermoplastic layer between CFRP and metal is shown. The CFRP-component is a $3 \mathrm{~K}$ plain weave with $30 \mathrm{vol} \%$ of carbon fiber embedded in an epoxy matrix and is made up of four layers $\left[0^{\circ} / 90^{\circ},+45^{\circ}\right]_{\mathrm{s}}$. The thermoplastic is a Polyphthalamide (PPA) with 30 vol\% of glass fiber and the metal is an EN AW-6082 (AISi1MgMn) aluminum. The complexity of this hybrid structure varies from a 2D- to a more complex 3D-geometry. In both cases interfaces, that are weak points in the structure [1], occur and have to be characterized by non-destructive testing methods.

\section{Thermography}

As thermography is a fast and widely used method to inspect different materials like metal [2] or CFRP [3], it is used for the characterization in this paper. Excitation sources are a flash light with $3.2 \mathrm{~kJ}$ and an eddy current coil for inductive testing. To record the heat waves in the samples an infrared camera with the bandwidth of $4,4 \mu \mathrm{m}-5,2 \mu \mathrm{m}$ and $145 \mathrm{fps}$ is utilized.

\subsection{Flash thermography}

The setup for the pulsed flash thermography is in reflection mode. The flash light heats up the surface and the heat wave diffuses into the sample. Defects act like thermal barriers, so areas with defects appear bright. Depending on time, defects in different depths can be detected. The temperature distribution in depth z can be described by Eq. (1):

$$
\mathrm{T}(\mathrm{z}, \mathrm{t})=\frac{Q}{\sqrt{\pi} \sqrt{\lambda \rho c} \sqrt{t}} \exp \left(-\frac{z^{2}}{4 \alpha t}\right)
$$

\subsection{Inductive thermography}

For inductive thermography, the eddy current coil is placed behind the sample. With this experimental setup the coil does not block the view on the sample. By calculating amplitude and phase images out of the raw data surface inhomogeneities can be eliminated. Depending on the modulation frequency $f$ the depth can be varied, see Eq. (2):

$$
\delta=\frac{1}{\sqrt{\pi f \mu \sigma}}
$$

\section{Results}

\subsection{Thermography for $2 D-s a m p l e s$}

Thermography results in figure 1 show that artificial induced defects like a pleat between the first and second layer can be detected with flash excitation as well as with inductive excitation. Additional the aluminum inlay and the fiber 
orientation in the first layer can be shown. Besides artificial defects, real defects that occur during mechanical testing are characterized in figure $1 \mathrm{c}$ ). It can be seen that around the aluminum there is a bright area which represents a delamination of CFRP from thermoplastic.

a)

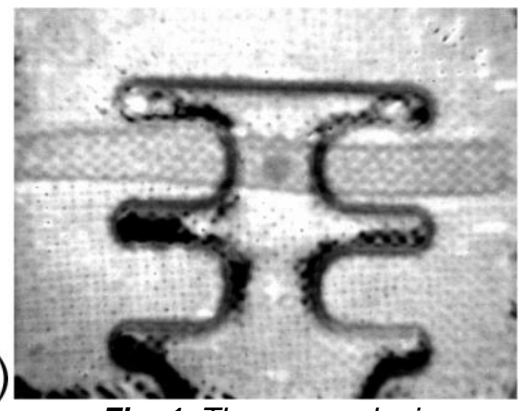

b)

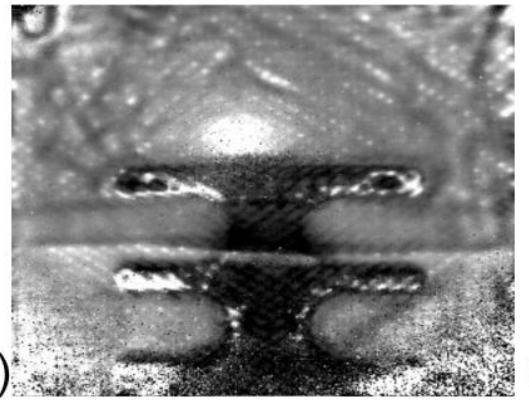

C)

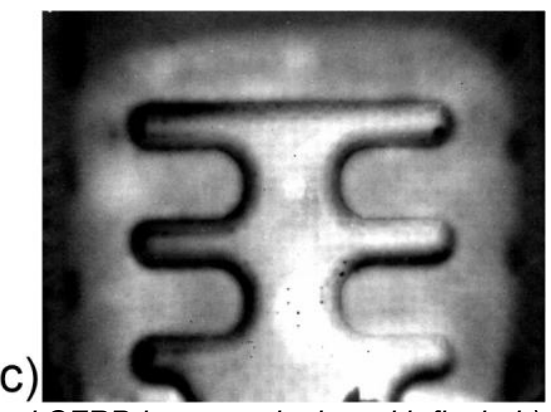

Fig. 1. Thermography images of a $2 D$-sample with a) a pleat in the second CFRP-layer, excitation with flash, b) inductive excitation and c) sample with a mechanically induced delamination

\subsection{Thermography for 3D-samples}

In contrast to the 2D-structure the 3D-hybrid has a curved CFRP-layer and a bent metal inlay. Figure 2 shows thermography images of this 3D-hybrid structure. Figure 2 a) and b) show a sample with a pleat between the first and second CFRP-layer. The pleat can clearly be seen above the metal inlay in the flash image as well as in the inductive image. Besides the pleat, the fiber orientation in the first layer and the metal inlay can be detected. Figure $2 \mathrm{c}$ ) shows a 3D-sample after mechanical testing. In the area of the metal inlay a bright area can be detected which can be interpreted as delamination.

a)

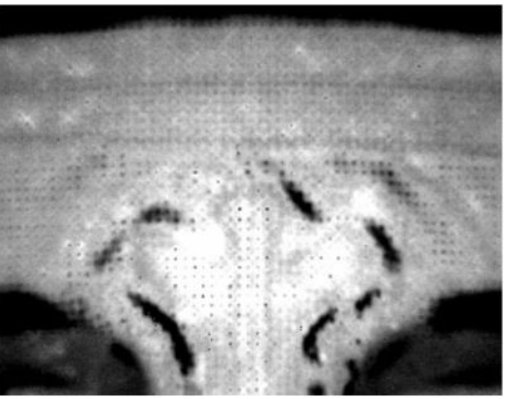

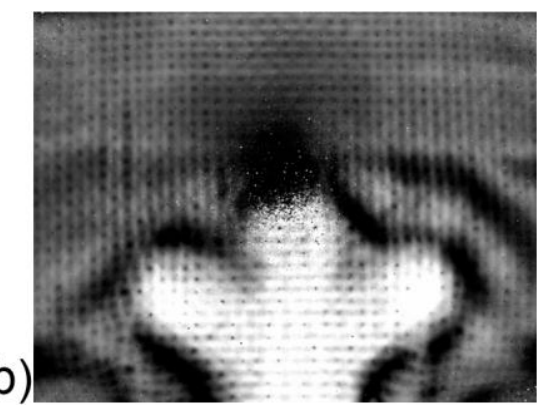

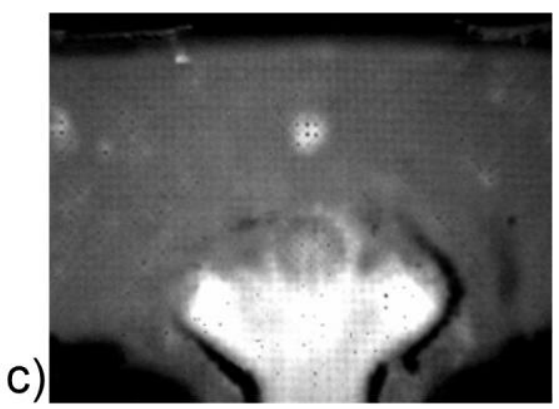

Fig. 2. Thermography images of a 3D-sample with a) a pleat in the second CFRP-layer - excitation with flash, b) inductive excitation and c) sample with a mechanically induced delamination

\section{Conclusions}

Active thermography with its excitation sources of flash and induction is very well suited to characterize CFRP and metal-CFRP hybrid structures. The results in this paper show that both artificial and mechanically induced defects can be detected. Despite the more complex geometry of the 3D-structure results for the detection of the pleat can be compared to the 2D-structure. Whereas the damage behavior of the mechanically induced delamination is different. For the 2Dstructure the delamination is around the whole metal inlay. In contrast, the delamination in the 3D-structure only arises in the immediate area of the CFRP/thermoplastic interface. The next step is to characterize the interface between aluminum and thermoplastic with electromagnetic acoustic transducer (EMAT).

\section{REFERENCES}

[1] Summa J., Schwarz M., Herrmann H.G., "Evaluating the Severity of defects in a metal to CFRP hybrid-joint with in situ passive thermography damage monitoring", Proceedings 5th International Conference on Integrity, Reliability \& Failure, IRF Porto, pp. 117-126, 2016.

[2] Park H., Choi M., Park J., Kim W., "A study on detection of micro-cracks in the dissimilar metal weld through ultrasound infrared thermography", Infrared Physics \& Technology 62, 124-131, 2014.

[3] Maierhofer C., Myrach P., Reischel M., Steinfurth H., Röllig M., Kunert M., "Characterizing damage in CFRP structures using flash thermography in reflection and transmission configurations", Composites B 57, 35-46, 2014. 